\title{
sFRP4 signalling of apoptosis and angiostasis uses nitric oxide-cGMP- permeability axis of endothelium
}

Uttara Saran", Mani Krishna Priya ${ }^{1}$, Uma Maheswari Balaguru ${ }^{1}$, Akila Swaminathan ${ }^{1}$, Shunmugam Nagarajan $^{1}$, Arun Dharmarajan ${ }^{3}$ and Suvro Chatterjee ${ }^{1,2 \#}$

${ }^{1}$ Vascular Biology Lab, AU-KBC Research Centre, Anna University, Chennai 600044, India

${ }^{2}$ Department of Biotechnology, Anna University, Chennai 600044, India

${ }^{3}$ Stem Cell and Cancer Biology Laboratory, School of Biomedical Sciences, Curtin Health Innovation Research Institute, Curtin University, Bentley, WA, Australia

\section{\# Corresponding author}

Dr. Suvro Chatterjee

Vascular Biology Lab,

AU-KBC Research Centre,

MIT Campus, Anna University,

Chennai 600 044, Tamil Nadu, India

Phone: +91 4422234885 ext. 48

Fax: $\quad+914422231034$

e-mail ID: soovro@yahoo.ca 


\begin{abstract}
Nitric oxide (NO) plays a critical role in endothelial functions such as cellular migration, vascular permeability and angiogenesis. Angiogenesis, the formation of new blood vessels from "pre-existing" ones is a carefully regulated process and essential during reproduction, development and wound healing. Previously our lab group reported that Secreted Frizzled-Related Protein 4 (sFRP4) could inhibit angiogenesis in both in vitro and in vivo conditions. sFRP4 belongs to a family of secreted glycoproteins that function as antagonists of the canonical Wnt signalling pathway. Although the pro-apoptotic role of sFRP4 is well discussed in literature, little is known in regards to its anti-angiogenic property. The objective of this study was to elucidate sFRP4 implications in NO biology of the endothelium. Results demonstrate that sFRP4 causes endothelial dysfunction by suppressing NO-cGMP signaling and elevating corresponding ROS levels. The imbalance between NO and ROS levels results in apoptosis and subsequent leakiness of endothelium as confirmed in vivo (Texas red/Annxin - CAM assay) and in vitro (Monolayer permeability assay) conditions. Furthermore utilizing peptides synthesized from the CRD domain of sFRP4, our results showed that while these peptides were able to cause endothelial dysfunctions, they did not cause apoptosis of the endothelial cells. Thereby confirming that sFRP4 can mediate its anti-angiogenic effect independent of its pro-apoptotic property. In conclusion, the current study reports that sFRP4-mediated anti-angiogenesis occurs as a result of impaired NO-cGMP signaling which in turn allow for elevation of redox levels and promotion of apoptosis of endothelial cells.
\end{abstract}

Key words: sFRP4, nitric oxide; endothelium, cGMP, reactive oxygen species, apoptosis 


\subsection{Introduction}

Secreted frizzled-related protein 4 (sFRP4) belongs to a family of secreted glycoproteins that function as one of the antagonists of the Wnt signaling pathway [1], which in turn plays an essential role in early development, cellular proliferation, migration and angiogenesis [2, 3]. sFRP4 has been reported to play a pro-apoptotic role during normal homeostasis of tissues including skin and mammary glands [4-9]. Its expression is frequently reported to be down-regulated or deleted in various malignancies resulting in the up-regulation of Wnt/ $\beta$-catenin signaling and excessive cell proliferation [10-16]. Subsequent restoration of sFRP4 expression has been shown to not only abrogate Wnt/ $\beta$-catenin mediated proliferation but also promote apoptosis of the cancer cells [10, 11, 17 ]. Furthermore sFRP4 expression has been shown to influence chemo-response of cancer cells where low expression is correlated with chemoresistance [18$20]$.

We were the first to demonstrate that in addition to its pro-apoptotic and anti-proliferative properties, sFRP4 is also a potent angiogenic inhibitor [21], by demonstrating that sFRP4 could inhibit proliferation and migration of endothelial cells (EC). Another important event, which defines angiogenesis, is endothelial permeability. It has been shown that the microvasculature is hyperpermeable to plasma proteins at the initial phase of angiogenesis. An intact endothelial monolayer demonstrates low-level "basal” permeability. Acute and short-term hyperpermeability, induced by vascular permeability factors such as VPF/VEGF-A and other vascular permeabilizing agents, define pro-angiogenic events by cross talking with small GTPases, cytokines and adherence junctions [22]. However in regards to the mechanism underlying the anti-angiogenic role of sFRP4, our previous studies were only able to hint that the anti-angiogenesis effect was mediated indirectly as a consequence sFRP4 suppression of nuclear translocation of $\beta$-catenin and elevated intracellular reactive oxygen species (ROS) induced apoptotic death. A preliminary finding of Muley et al [21] was that administration of exogenous VEGF was unable to blunt sFRP4 mediated effects, however the effect of sFRP4 on the downstream nitric oxide (NO) pathway remains unknown. Considering that an increase in ROS production and the corresponding inactivation of NO has been shown to cause endothelial dysfunction [23], we hypothesized that sFRP4 interferes with endothelial permeability via NO/sGC/cGMP pathway and thereby perturbs angiogenesis.

The present study elaborated that ectopic dosage of sFRP4 induces oxidative stress in endothelium and simultaneously attenuates NO production. This imbalance in redox status of endothelium promotes apoptosis; and facilitates para-cellular gap formation in endothelium. The endothelial monolayer with para-cellular gaps and pro-apoptotic stress pro-creates an ideal ambiance for anti-angiogenic milieu in the endothelium. 


\section{Materials and Methods}

\subsection{Materials}

DMEM (Dulbecco's modified Eagle’s medium) was purchased from PAN-Biotech GmbH. FBS (Fetal bovine serum) was purchased from Invitrogen Life Technologies. Spermine NONOate (SP) and guanosine-3' 5'-monophosphate 8-bromo-sodiumsalt (8-Br) were purchased from EMD Biosciences, Inc., California, USA. Diaminorhodamine (DAR-4M-AM), sildenafil citrate (SC), 3-(5-hydroxymethyl-2furyl)-1-benzyl indazole (YC-1), vascular endothelial growth factor (VEGF), L-NAME, Amplex Red and Catalase (CAT) from bovine liver were obtained from Sigma Chemical Co., St. Louis. Phalloidin-Alexa Fluor 568 (Phalloidin), DAR-4M AM, diamino-fluorescein (DAF-FM), 2', 7'-dichlorofluorescein diacetate (DCF-2DA) and Dihydrorhodamine 123 (DHR) was purchased from Molecular probe, Oregon,USA. sFRP4 protein was purchased from Sino Biological Inc. sFRP4 CRD peptides were obtained from Imgenex India Pvt. Ltd. Bhubaneshwar, India. Cadmium chloride $\left(\mathrm{CdCl}_{2}\right)$ was purchased from Hi-Media, Mumbai, India. All other chemicals were of reagent grade and were obtained commercially.

\subsection{Cell Lines}

Immortalized endothelial hybrid cell lines, EAhy926 and ECV304 cells stably transfected with eNOSGFP constructs (kind gifts from Dr. C.J.S Edgell and Dr. Vijay Shah, Mayo Clinic Rochester USA) and primary dermal microvascular endothelial cells (HDMVEC; from ATCC, USA) were cultured in DMEM supplemented with 10\% FBS and 1\% penicillin-streptomycin.

\subsection{Estimation of Nitric oxide (NO):}

2.3.1. Griess assay: NO was measured using Griess protocol as previously described [24]. Griess assays were performed on EAhy926 cells to determine optimal dosage (125, 250 and $500 \mathrm{pg} / \mathrm{ml}$ ) and duration (30 min, 1, 3, 6, 12 and 24 h) of sFRP4 treatments. The Griess assay was also performed on freshly slaughtered bovine aortas obtained from government authorized abattoir. Briefly, bovine aortas were washed with 1 X PBS and then cut into ring like structures. Aortic rings were then placed in sterile 12well petri-plate and incubated with PBS containing either sFRP4, positive control L-Arginine (L-Arg) or negative control L-NAME for $4 \mathrm{~h}$. The solution was then carefully collected and measured.

2.3.2. NO measurement using DAF-2DA: DAF-2DA was performed on HDMVEC and EAhy926 cells following treatment with $250 \mathrm{pg} / \mathrm{ml}$ sFRP4 for $4 \mathrm{~h}$ as per the protocol described elsewhere [25]. 
2.3.3. DAF-FM fluorimetric assay: DAF-FM was performed on EAhy926 cells treated with sFRP4 (250 $\mathrm{pg} / \mathrm{ml})$, Bradykinin (BK; $5 \mu \mathrm{M})$, SP $(10 \mu \mathrm{M}), 8-\mathrm{Br}(50 \mu \mathrm{M})$, CAT (30 U), MnTBAP $(10 \mu \mathrm{M})$ and their combinations with sFRP4 for $4 \mathrm{~h}$ as per the protocol described elsewhere [26]. L-Arg was used as positive control. DAF-FM were also performed for sFRP4 CRD domain peptides (CP-128-10, CP-12910, CP-130-10, CP-131-10, CP-132-10, CP-133-10, CP-134-10).

2.3.4. DAR-4M-AM live cell imaging: ECV304 stably transfected with eNOS-GFP were then grown overnight as monolayers on coverslips. The monolayers were treated with sFRP4 for $4 \mathrm{~h}$. Following treatment the monolayers were washed with $1 \mathrm{X}$ PBS and then incubated with NO-specific fluorescence probe DAR-4M-AM for 10 min. The monolayer was washed again with 1X PBS before corresponding bright field and fluorescence images were taken at 20X magnification using an Olympus fluorescence microscope. The fluorescence intensity of eNOS and DAR were calculated using Adobe Photoshop version 7.0 software program.

2.4. Fluorescence live cell imaging: ECV304 stably transfected with eNOS-GFP cells were grown overnight at $40 \%$ confluency on coverslips in 12-well plates. The cells were treated with control, sFRP4, BK and sFRP4 + BK respectively. Following treatment, cells were placed in live-cell chambers and eNOS localization was observed using an Olympus IX71 epifluorescence microscopy system equipped 209 with a DP71 camera.

2.5 Phallodian staining: EAhy926 monolayers and single cell plated on coverslips were treated with sFRP4 for $4 \mathrm{~h}$ before being stained with phallodian as per the protocol described elsewhere [27].

\subsection{Permeability assays}

The term 'leakiness' denotes net permeability and is inclusive of both para-cellular and trans-cellular vascular solute fluxes [27].

2.6.1 Texas Red monolayer permeability: Endothelial monolayer leakiness was assessed as previously described [27], with minor modifications (Figure 1 C). Briefly, EAhy926 monolayers were grown overnight on collagen-coated polycarbonate membranes in permeability chambers. Monolayers were treated with sFRP4, SP, 8-Br, CAT, MnTBAP and their combinations with sFRP4 for 4 h. Following treatment, the monolayers were gently washed with $1 \mathrm{X}$ PBS and then incubated with $10 \mu \mathrm{M}$ Texas red for $1 \mathrm{~h}$. The solution from the lower chamber was then collected and measured using a Varian Cary Eclipse UV-Vis Fluorescence spectrophotometer at excitation/emission of 589/615 nm. Texas red monolayer permeability assays were also performed following treatment with sFRP4 CRD domain peptides CP-13310 and CP-134-10. 
2.6.2. Texas red permeability assay in Chorionic-Allantoic Membrane (CAM): Fertilized four day incubated eggs were obtained from Poultry Research Station, Potheri, Chennai. Texas red permeability assays were performed on CAM treated with control (1X PBS), sFRP4, SP and sFRP4 + SP for 4 h as per protocol described elsewhere [26].

2.6.3. $\mathrm{CdCl}_{2}$ Texas red curve: A Texas Red-Cadmium scale was generated by treating EC monolayers and CAMs to increasing doses of $\mathrm{CdCl}_{2}(0-100 \mu \mathrm{M} / \mathrm{ml})$ for $2 \mathrm{~h}$.

2.7. Transfections: EAhy926 cells grown in 24-well plates were transfected with FlincG plasmid (addgene-pcDNA3.1 (+)-deltaFlincG) encoding PKG-GFP as per the protocol described previously [25]. $40 \mathrm{~h}$ post transfection, cells were treated with sFRP4 and negative control cPTIO for $4 \mathrm{~h}$. Images were taken using an Olympus IX71 fluorescence microscope and the fluorescence intensity was calculated using the Adobe Photoshop version 7.0 software programe.

2.8. Endothelial scratch-wound assay: The scratch-wound assay was performed on EAhy926 monolayers treated with sFRP4, YC-1, SC and 8-Br and their combinations as previously described [25]. Images of the wound area were taken 0, 2 and 4 hours using a Nikon digital camera and the area of the cell-free wound at 2 and $4 \mathrm{~h}$ time points was analyzed using Image $\mathrm{J}$ image software.

2.9. Western blot analysis: EAhy926 cells were treated with sFRP4 for 4 h. Triton X soluble and insoluble fractions were prepared as per protocol described elsewhere [23] and western blots were performed for eNOS protein expression (1:1000 dilution, SC-654, 199 Santa Cruz, CA, USA).

2.10. Estimation of reactive oxygen species (ROS): Four parameters were assessed including total ROS, superoxide, peroxynitrite, and hydrogen peroxide $\left(\mathrm{H}_{2} \mathrm{O}_{2}\right)$ production in EAhy926 endothelial cells following respective treatments.

2.10.1. Total ROS assay: In first experiment, cells were treated with sFRP4 doses in the presence and absence of superoxide dismutase (SOD) (150 SI Units). In the second experiment cells were treated with sFRP4, SP, 8-Br, CAT, MnTBAP and their respective combinations. Total ROS levels were measured using fluorescent probe DCF-DA as per the protocol described elsewhere [21].

2.10.2. Amplex red assay: Cells were treated with sFRP4, SP, 8-Br, CAT, MnTBAP and their combinations for $4 \mathrm{~h}$. The Amplex red assay was performed as per protocol previously described [21].

2.10.3. Measurement of peroxynitrite: Following treatment with SFRP4, SP, 8-Br, CAT, MnTBAP and their combinations for $4 \mathrm{~h}$, peroxynitrite levels were measured using DHR as described elsewhere [28]. 
2.10.4. Nitro Blue Tetrazolium (NBT) Assay: Following treatment with sFRP4, SP, 8-Br, CAT, MnTBAP and their combinations for $4 \mathrm{~h}$, the NBT assay was performed as per protocol described previously [21].

2.11. Measurement of Annexin-Propidium iodide staining: Apoptosis was detected in EAhy926 cells and CAM with help of Annexin-propidium iodide (PI) staining (Calbiochem PF032). EC were subjected to treatment with sFRP4, SP, 8-Br, CAT, MnTBAP and their combinations for $4 \mathrm{~h}$. Staurosporine (STP) was used as positive control. After treatment, the cells were then washed three times with $1 \mathrm{X}$ PBS and then incubated with binding buffer, Annexin V and PI, respectively, as per the manufacture's protocol. Fluorescent images of Annexin and PI were captured using Olympus XL70 fluorescent microscope. The fluorescence intensity of the images was quantified using Adobe Photoshop version 7.0. Annexin-PI staining was also performed on EC following treatment with sFRP4 CRD domain peptides CP-133-10 and CP-134-10.

The same protocol was applied for CAM following treatment with sFRP4, SP and their combination, albeit with minor modification. Briefly, following treatment, CAM were washed with $1 \mathrm{X}$ PBS and incubated with Texas red dye for $30 \mathrm{~min}$. The CAM were again washed with 1X PBS and incubated with Annexin V + binding buffer alone. The CAM were then fixed with 4\% paraformaldehyde for $10 \mathrm{~min}$ before images in bright field, green (Annexin V) and red (Texas Red) fluorescence were taken using an Olumpus IX71 epifluorescence microscope equipped with a DP71 camera.

2.12. Statistical analysis: All experiments were performed in triplicate $(n=3)$ unless specified otherwise. Data are presented as mean \pm SE. Data was analyzed using one-way analysis of variance (ANOVA), student's t test, and Turkey's post hoc tests using the SIGMA STAT software package version 4. $\mathrm{p}<0.005$ was considered to be statistically significant.

\section{Results}

\subsection{SFRP4 induced leakiness of endothelium}

The accumulation of Texas Red dye within CAM blood vessels is indicative of vessel leakiness. Analysis of Texas Red permeability assays performed on CAM showed that sFRP4 treatment ( $250 \mathrm{pg} / \mathrm{ml})$ induced significant accumulation of dye within blood vessels when compared to control vessels $(\mathrm{p}<0.001)$ (Figure $1 \mathrm{~A}$ and B). Furthermore sFRP4 demonstrated a dose dependent effect on EC leakiness in vitro, with both the 250 and $500 \mathrm{pg} / \mathrm{ml}$ doses of sFRP4 inducing significant dye permeation $(\mathrm{p}<0.001)$ past the EC 
monolayer in comparison to control monolayers (Figure 1D). As both doses yielded similar results, we elected to use $250 \mathrm{pg} / \mathrm{ml}$ as our treatment dose for subsequent experiments. Although the $125 \mathrm{pg} / \mathrm{ml}$ dose of sFRP4 also showed increased dye permeation into the lower chamber of the permeability chamber apparatus, this finding was not significant. In order to estimate the extent of sFRP4 mediated endothelial leakiness in both in vivo (CAM) and in vitro (EC monolayer) conditions, we plotted our results on a Texas Red-Cadmium scale. Cadmium is a well known teratogen that causes endothelial leakiness [29, 30]. The Texas Red-Cadmium scale was generated by subjecting CAM and EC monolayers to increasing concentrations of $\mathrm{CdCl}_{2}$ thereby resulting in an increasing scale of dye permeation. When plotted on the Cadmium scale, results demonstrated that $250 \mathrm{pg} / \mathrm{ml}$ dose of sFRP4 induced endothelial leakiness that was comparable to $5 \mu \mathrm{M} \mathrm{CdCl}_{2}$ (Figures $1 \mathrm{E}$ and $\mathrm{F}$ ). Assessment of cytoskeleton arrangements of EC using phallodin staining showed formation of large gaps in the EC monolayers following sFRP4 treatment which were absent in control monolayers (Figure 1G - monolayer). Furthermore phallodin staining of single cells revealed that the sFRP4 treated cells demonstrated diffused and diminished actin distribution instead of the rigid and even actin distribution observed in control cells (Figure 1G - single cells; Supplementary Figure 1A).

\section{2. sFRP4 attenuates NO-cGMP signaling in endothelial cells}

To determine if sFRP4 inhibition of endothelial function occurred via suppression of NO pathway, Griess and DAF-FM assays were first used to assess the dose dependent effect of sFRP4 on NO production of EC. L-Arg a precursor of NO was used as positive control. Results showed that all three doses of sFRP4 (125, 250 and $500 \mathrm{pg} / \mathrm{ml}$ ) significantly decreased NO production of EC, when compared to control cells, (Figure 2A). Furthermore, a time dependent study showed that $250 \mathrm{pg} / \mathrm{ml}$ of sFRP4 began inhibiting endothelial NO production around $3 \mathrm{~h}$ post treatment and continued to significantly inhibit NO production for $24 \mathrm{~h}$ (Figure 2B). Next using DAF-2DA imaging, we compared the effects of sFRP4 on NO production between an immortalized hybrid endothelial cell line EAhy926and a primary endothelial cell line HDMVEC. Results showed that SFRP4 significantly decreased NO production EC irrespective of cell origin (Figure 2C). Additionally sFRP4 also significantly $(\mathrm{p}<0.001)$ decreased NO production of endothelial cells lining freshly dissected bovine aortic rings (Figure 2D).

We then investigated the effects of sFRP4 on eNOS localization with the help of ECV304 cells that were stably transfected with eNOS-GFP. Results showed that treatment with sFRP4 induced extensive eNOS re-localization from nuclear membrane towards plasma membrane of the cells (depicted with white arrows) in sFRP4 treated cells, compared to control and BK treated cells (Figure 3A). Interestingly subsequent addition of BK to sFRP4 treated cells was able to partially restore eNOS localization to the 
nuclear membrane from the plasma membrane (depicted with yellow arrows) (Figure 3A). Western blot analysis of eNOS in triton-soluble and triton-insoluble fractions, showed increased eNOS in tritoninsoluble fractions in sFRP4 treated cells when compared to control cells (Figure 3B), hinting that sFRP4 inhibition of NO could be occurring via the sGC-cGMP signaling pathway.

To establish that SFRP4 has a direct effect on downstream NO-cGMP signaling, we demonstrated that on sFRP4 treatment of FlincG (a PKG-GFP construct) transfected EC significantly decreased cellular cGMP level when compared to control transfected cells ( $\mathrm{p}<0.001)$ (Figures 3C). Interestingly the sFRP4 induced effects were similar to the effect of the negative control cPTIO (a NO specific quencher). Scratch-wound assays showed that addition of activators of sGC-cGMP signaling like YC-1, SC were able to alleviate sFRP4 mediated inhibition of wound healing, with cGMP analog 8-Br demonstrating significant recovery compared to sFRP4 treated cells (Figure 4A). Furthermore supplementation of NO either via 8-Br or SP was able to significantly blunt sFRP4 effects and restore NO production of treated cells $(\mathrm{p}<0.001)$ (Figure 4B). Taken together results confirm that sFRP4 inhibition of NOS attenuates cGMP production and therefore dampens cGMP-PKG signalling in endothelial cells.

\subsection{Rescuing endothelial cells from sFRP4 mediated effect by reducing ROS levels}

In the current study we have demonstrated that $250 \mathrm{pg} / \mathrm{ml}$ sFRP4 significantly elevated the hydrogen peroxide, peroxynitrite and total ROS levels of EC (Supplementary Figure 1 B and C), while addition of anti-oxidant SOD was able to significantly decrease total ROS levels of these cells. Although addition of both CAT (an enzyme that activates hydrogen peroxide degradation) and MnTBAP (SOD) to sFRP4 treated EC demonstrated increased NO levels, this finding was not significant (Figure 4C). Subsequent addition of either antioxidants or NO donors (SP) or cGMP analog 8-Br significantly decreased redox levels (total ROS, hydrogen peroxide, peoxynitrite and superoxide) of sFRP4 treated EC when compared to cells treated with only sFRP4 (Figure 5). Elevated ROS levels are associated with increased apoptosis. Accordingly, Annexin/PI staining of EC showed significantly increased annexin positive cells, indicative of apoptosis. In contrast, administration of NO or negating ROS levels was found to rescue the cells from apoptosis (Figure 6; Supplementary Figure 1 D).

\section{4. sFRP4 mediates its anti-angiogenic effect by dampening NO-cGMP signalling}

In the current study we demonstrate that sFRP4 directly dampens NO production in EC. To determine if decreased NO production, rather than elevated ROS/apoptosis, was responsible for the anti-angiogenic effects of sFRP4, we performed the following three separate experiments. 
In the first experiment, we assessed real time NO production and cell morphology of stably transfected ECV304 cells following treatment with sFRP4. eNOS expression (green) and NO diffusion from the cells (red) was recorded for both control and sFRP4 treated monolayer (Figure 7A). Control monolayers exhibited a greater proportion of eNOS expressing cells, larger distribution of diffused NO, as indicative of DAR-4M-AM florescence, and structurally robust cells with no gaps (Figure 7B). Additionally merged images of eNOS and DAR-4M-AM images showed significantly higher number of yellow cells. In contrast sFRP4 treated monolayers showed significantly decreased eNOS expression and consequently reduced NO diffusion $(\mathrm{p}<0.001)$ (Figure $7 \mathrm{C})$. Similarly merging of images revealed very a weak proportion of yellow cells. The most significant observation was that the decreased NO production seemed to directly correlate with an increased number of gaps in the EC monolayer.

In the second experiment, we co-performed Texas red and Annexin staining on sFRP4 treated CAM blood vessels. Interestingly results showed that areas demonstrating increased Texas red accumulation within the blood vessels following sFRP4 treatment also showed increased apoptosis when compared to control and SP treated CAMs. Subsequent addition of the NO donor SP to sFRP4 treated CAMs was able to significantly curtail the amount of dye permeating into the vessel despite not being able to completely inhibit apoptosis (Figure 8 A-C). Further analysis of in vitro permeability assays revealed that restoration of NO levels using either SP or 8-Br was able to significantly decrease gap formation of sFRP4 treated EC monolayers. Whereas neutralization of ROS levels by anti-oxidants (CAT/MnTBAP) were not able to prevent sFRP4 mediated leakiness of the EC monolayer (Figure 8D).

Finally with the help of synthesized peptides of the CRD domain of sFRP4, we determined if sFRP4 could mediate its anti-angiogenic role independent of its pro-apoptotic effect. Of the seven peptides available, we identified peptides CP-131-10, CP-133-10, CP-134-10 were able to significantly decrease NO production in EC cells (Figure 9A). While both peptide CP-133-10 and CP-134-10 were able to cause significant leakiness of the EC monolayer (albeit to a lesser degree than whole sFRP4 protein) compared to control cells; neither peptide demonstrated any significant increase in apoptosis (Figure 9 B-D). Taken together these findings confirm that sFRP4 can mediate its anti-angiogenic effect via inhibition of NO signaling and endothelial dysregulation in addition to its ROS-induced apoptosis. We also confirm that sFRP4 can implement its anti-angiogenic effect independent of its pro-apoptotic role.

\section{Discussion}

The discovery of sFRP4 anti-angiogenic properties was particularly profound. sFRP4 mimicked the efficacy demonstrated by the clinically approved angiogenesis inhibitor, Avastin (a VEGF-blocking antibody) in inhibiting angiogenesis of aggressive ovarian tumors implanted in BALB/c nude mice [21]. 
A limitation of this study was that the combined effect of sFRP4 and Avastin was not explored, however preliminary in vitro data showed that exogenous VEGF was unable to negate sFRP4's inhibitory effects. Consequently, in part due to the finding that apoptotic activity in these tumors was localized to endothelial regions, it was postulated that the anti-angiogenic property of sFRP4 possibly occurred as an indirect consequence of its anti-proliferative and pro-apoptotic effects. The present study made an effort to understand the link between the anti-angiogenesis and pro-apoptotic effects of sFRP4 on endothelial cells.

Sinha et al [31] highlighted the role played by NO/sGC/cGMP pathway during endothelial ring formation, a crucial angiogenic step. Interestingly studies demonstrating the failure of VEGF in inducing angiogenesis in $\mathrm{eNOS}^{-/-}$mice, indicate that NO is the critical mediator of VEGF induced angiogenesis [32, 33]. Maintenance of the endothelium and the regulation of vascular permeability is also a key function of the NO/cGMP pathway [27, 33, 34]. Considering the importance of NO in endothelial biology, we postulated that SFRP4 mediates its anti-angiogenic ability via direct inhibition of NO within the endothelial cell, disrupting NO signaling, which causes subsequent endothelial dysfunction and leakiness of the endothelium. Preliminary results indicate that sFRP4 treatment increased endothelial leakiness in both in vitro and in vivo conditions. We were able to attribute sFRP4 induced endothelial leakiness to the formation of gaps resulting from sFRP4 mediated reorganization of the EC cytoskeleton. Furthermore, using live cell imaging, we were able to positively correlate sFRP4 mediated attenuation of endogenous NO production and the subsequent formation of gaps in the endothelial monolayer. Results also demonstrated that SFRP4 decreased NO production in EC irrespective of cell origin (micro- vs. macro-vascular EC) and that this inhibition remained effective up to $24 \mathrm{~h}$ post treatment. Our data parallels previous studies that have similarly shown altered endothelial actin cytoskeleton and increased vascular permeability following inhibition of NO synthesis [26, 27, 35].

The management of eNOS sub-cellular localization is key to the proper facilitation of NO production, while its mislocalization impairs angiogenesis [36, 37]. Live cell imaging revealed that sFRP4 prevented the peri-nuclear localization of eNOS. This was further confirmed by immunoblot analysis which showed that SFRP4 treatment increased eNOS content in the triton-insoluble fraction, a known abode for caveolin, a chronic inhibitor of eNOS activity. Based on these results we postulated that sFRP4 modulates the subcellular localization of eNOS to blunt NO production and endothelial permeability. Recently Perumal et al [39] reported that sFRP4 induced cell death via its inhibition of Akt survival pathways. Considering that direct phosphorylation of eNOS by Akt is one of the methods via which VEGF facilitates the activation of eNOS, it is quite plausible that sFRP4 can target eNOS both directly as well as indirectly, 
which also explains why exogenous VEGF was ineffective against sFRP4. To further dissect sFRP4 inhibition of NO signaling, we next probed the NO/sGC/cGMP pathway. NO once released binds to sGC to produce cGMP [39-42]. To directly assess the effect of sFRP4 on cGMP signaling, we transfected EAhy926 cells with a PKG-GFP construct (FlincG) prior to treatment with sFRP4. Results demonstrated that sFRP4 significantly suppressed cellular cGMP level, suggesting that sFRP4 induces endothelial leakiness in a sGC-cGMP dependent manner.

Omori \& Kotera et al [43] demonstrated that the inhibition of in vitro tube formation in $\mathrm{eNOS}^{-/-}$mice could be restored via addition of a NO donor. While Antonova et al [44] reported that preconditioning with low doses of a NO donor could accelerate the repair and maintenance of endothelial integrity via the sGC pathway. Previous reports from our lab group have also shown that treatment with NO donors or cGMP analogs can restore and protect endothelial function, morphology and barrier integrity [26, 27, 4547]. Similarly, we noted that addition of NO donor SP or activators of NO/sGC/cGMP activity such as YC-1 (an NO independent activator of sGC), SC (which increases cellular cGMP by PDE inhibition) and 8-Br (cGMP analogue) could blunt sFRP4 mediated effects. Previously we have shown that endothelial dysfunction, whether hypoxia-driven or induced by teratogens (cadmium, thalidomide), occurs primarily as a consequence of decreased intercellular NO bioavailability and/or bioactivity and elevated ROS levels, which promotes apoptosis [26, 27, 45-47]. Superoxide production in EC has been reported to contribute to enhanced permeability of EC membrane [48], particularly as NO is known for its strong affinity to react with superoxide radicals [49] to form peroxynitirite and thereby causing leakiness of the endothelium. Alternatively pretreatment of cells with NO donors has been shown to increase endothelial cGMP activity and suppress hydrogen peroxide-related effects on permeability [50]. Similarly, addition of both the NO donor, SP and the cGMP analog, 8-Br were effective in both suppressing ROS levels and preventing apoptosis in sFRP4 treated cells. Furthermore, we observed that both SP/8-Br cGMP treatments were able to blunt sFRP4 mediated effects (NO dampening, elevation of ROS/apoptosis) with greater efficacy when compared to antioxidant treatment.

In vivo co-localization of annexin and texas red staining revealed that sFRP4 mediated its anti-angiogenic effects by both inhibition of NO signaling as well as causing apoptosis of the affected cells. Intriguingly, addition of SP to SFRP4 treated CAMs was able to significantly restore endothelial function and decrease the permeability of these vessels, despite not having completely inhibited apoptosis of these cells. This evidence, coupled with the ineffectiveness of antioxidants to blunt sFRP4 mediated leakiness of the EC monolayer, indicates that the restoration of NO, rather than the neutralization of ROS, attenuates leakiness of the endothelium. This finding also raised the question whether the anti-angiogenic and pro- 
apoptotic roles of sFRP4 could function independent of one another. We tested this theory by studying the effects of peptides synthesized from the sFRP4 CRD domain, which has been previously reported by Longman et al [51] to be the domain responsible for sFRP4's anti-angiogenic affect. Results showed that while peptides 6 and 7 were able to decrease NO production and cause significant leakiness of the EC monolayer, they did not induce these cells to undergo apoptosis like the total protein, sFRP4 could.

The present study reveals that sFRP4 mediates its anti-angiogenic effects, in part, via the direct inhibition of the NO/cGMP signaling pathway, resulting in endothelial dysregulation. Furthermore this study also demonstrates that the anti-angiogenic and pro-apoptotic roles of this protein can target endothelial cells simultaneously. More importantly, sFRP4 appears to induce apoptosis in the endothelium via cGMP inhibition, and the manifestation of apoptosis in the endothelium is initially leakiness of the monolayer followed by disruption of vascular structures. In conclusion, sFRP4 uses two distinct pathways in the endothelium; pro-apoptotic and anti-angiogenesis, by dampening the NO-cGMP-permeability cascade in endothelial cells.

\section{Conflict of interest}

The authors declare that there are no conflicts of interest.

\section{Acknowledgements}

This work was financially supported by Department of Biotechnology, Government of India (Project No: BT/PR11770/BRB/10/687/2009) and University Grant Commission-Faculty Recharge Programme (UGCFRP), Government of India to SC.

\section{References}

[1] Kawano Y, Kypta R: Secreted antagonists of the Wnt signalling pathway. J Cell Sci (2003) 116:2627-2634

[2]Clevers H, Nusse R. Wnt/ $\beta$-catenin signaling and disease. Cell (2012) 149:1192-205.

[3]Wang J, Sinha T, Wynshaw-Boris A. Wnt signaling in mammalian development: lessons from mouse genetics. Cold Spring Harb Perspect Biol (2012) 4:a007963.

[4] James, I. E., Kumar, S., Barnes, M. R., Gress, C. J., Hand, A. T., Dodds, R. A., Connor, J. R., Bradley, B. R., Campbell, D. A., Grabill, S. E., Williams, K., Blake, S. M., Gowen, M. \& Lark, M. W.. FrzB-2: a human secreted frizzled-related protein with a potential role in chondrocyte apoptosis. Osteoarthritis Cartilage (2000) 8, 452-63.

[5]Schumann, H., Holtz, J., Zerkowski, H. R. \& Hatzfeld, M.. Expression of secreted frizzled related proteins 3 and 4 in human ventricular myocardium correlates with apoptosis related gene expression. Cardiovasc Res, (2000) 45, 720-8. 
[6] Berndt, T., Craig, T. A., Bowe, A. E., Vassiliadis, J., Reczek, D., Finnegan, R., Jan De Beur, S. M., Schiavi, S. C. \& Kumar, R.. Secreted frizzled-related protein 4 is a potent tumor-derived phosphaturic agent. J Clin Invest, (2003)112, 785-94.

[7] Lacher, M. D., Siegenthaler, A., Jager, R., Yan, X., Hett, S., Xuan, L., Saurer, S., Lareu, R. R., Dharmarajan, A. M. \& Friis, R.. Role of DDC-4/sFRP-4, a secreted frizzledrelated protein, at the onset of apoptosis in mammary involution. Cell Death Differ (2003) 10, 528-38.

[8]Maganga, R., Giles, N., Adcroft, K., Unni, A., Keeney, D., Wood, F., Fear, M. \& Dharmarajan, A. Secreted Frizzled related protein-4 (sFRP4) promotes epidermal differentiation and apoptosis. Biochem Biophys Res Commun (2008) 377, 606-11.

[9]Constantinou, T., Baumann, F., Lacher, M. D., Saurer, S., Friis, R. \& Dharmarajan, A. SFRP-4 abrogates Wnt-3a-induced beta-catenin and Akt/PKB signalling and reverses a Wnt-3a-imposed inhibition of in vitro mammary differentiation. J Mol Signal (2008) 3, 10.

[10]Lee, A. Y., He, B., You, L., Dadfarmay, S., Xu, Z., Mazieres, J., Mikami, I., Mccormick, F. \& Jablons, D. M.. Expression of the secreted frizzled-related protein gene family is downregulated in human mesothelioma. Oncogene (2004) 23, 6672-6.

[11]He, B., Lee, A. Y., Dadfarmay, S., You, L., Xu, Z., Reguart, N., Mazieres, J., Mikami, I., Mccormick, F. \& Jablons, D. M. Secreted frizzled-related protein 4 is silenced by hypermethylation and induces apoptosis in beta-catenin-deficient human mesothelioma cells. Cancer Res (2005) 65, 743-8.

[12]Zou, H., Molina, J. R., Harrington, J. J., Osborn, N. K., Klatt, K. K., Romero, Y., Burgart, L. J. \& Ahlquist, D. A. Aberrant methylation of secreted frizzled-related protein genes in esophageal adenocarcinoma and Barrett's esophagus. Int J Cancer (2005) 116, 584-91.

[13]Qi, J., Zhu, Y. Q., Luo, J. \& Tao, W. H. Hypermethylation and expression regulation of secreted frizzled-related protein genes in colorectal tumor. World J Gastroenterol (2006) 12, 7113-7.

[14]Carmon, K. S. \& Loose, D. S. Secreted frizzled-related protein 4 regulates two Wnt7a signaling pathways and inhibits proliferation in endometrial cancer cells. Mol Cancer Res (2008) 6, 1017-28.

[15] Kohno H, Amatya VJ, Takeshima Y, Kushitani K, Hattori N, Kohno N, et al. Aberrant promoter methylation of WIF-1 and SFRP1, 2, 4 genes in mesothelioma. OncolRep (2010) 24:423-31.

[16] Jacob F, Ukegjini K, Nixdorf S, et al. Loss of secreted frizzled related protein 4 correlates with an aggressive phenotype and predicts poor outcome in ovarian cancer patients. PLoS One (2012) 7(2):e31885.

[17] Horvath, L. G., Lelliott, J. E., Kench, J. G., Lee, C. S., Williams, E. D., Saunders, D. N., Grygiel, J. J., Sutherland, R. L. \& Henshall, S. M. Secreted frizzled-related protein 4 inhibits proliferation and metastatic potential in prostate cancer. Prostate,(2007) 67, 1081-90.

[18] Saran U, Arfuso F., Zeps N.,. Dharmarajan A, Secreted frizzled-related protein 4 expression is positively associated with responsiveness to cisplatin of ovarian cancer cell lines in 
vitro and with lower tumour grade in mucinous ovarian cancers, BMC Cell Biol. 13 (2012) 25.

[19] S.Warrier, S.K. Balu, A.P. Kumar, M. Millward, A. Dharmarajan, Wnt antagonist, secreted frizzled-relatedprotein4(sFRP4),increases chemotherapeutic response of glioma stem-like cells,Oncol.Res.21(2013)93-102.

[20] S.Warrier, G.Bhuvanalakshmi, F.Arfuso, G.Rajan,M.Millward, A.Dharmarajan, Cancer stem-like cells from head and neck cancers are chemosensitized by the Wntantagonist, sFRP4, by inducing apoptosis, decreasing stemness, drug resistance and epithelial to mesenchymal transition, Cancer Gene Ther.21(2014)381-388

[21] Muley, A., Majumder, S., Kolluru, G. K., Parkinson, S., Viola, H., Hool, L., Arfuso, F., Ganss, R., Dharmarajan, A. \& Chatterjee, S. Secreted frizzled-related protein 4: an angiogenesis inhibitor. Am J Pathol, (2010) 176, 1505-16.

[22] Huber, O., C. Bierkamp, and R. Kemler. Cadherins and catenins in development. Curr. Opin. Cell Biol. Curr Opin Cell Biol. (1996) 5:685-91.

[23] G.K. Kolluru et al. / Nitric Oxide 22 (2010) 304-315

[24] Nims RW, Cook JC, Krishna MC, Christodoulou D, Poore CM, Miles AM, Grisham MB, Wink DA. Colorimetric assays for nitric oxide and nitrogen oxide species formed from nitric oxide stock solutions and donor compounds. Methods Enzymol. (1996) 268: 93105.

[25] Gajalakshmi P, Priya MK, Pradeep T, Behera J, Muthumani K, Madhuwanti S, Saran U, Chatterjee S. Breast cancer drugs dampen vascular functions by interfering with nitric oxide signaling in endothelium. Toxicol Appl Pharmacol. (2013) 1;269(2):121-31.

[26] Nagarajan, S., Rajendran, S., Saran, U., Priya, M. K., Swaminathan,A., Siamwala, J. H., Sinha, S., Veeriah, V., Sonar, P., Jadhav, V.,et al. Nitric oxide protects endothelium from cadmium mediated leakiness. Cell Biol. Int. (2013) 37, 495-506.

[27] Kolluru GK, Tamilarasan KP, Rajkumar AS, Geetha Priya S, Rajaram M, Saleem NK, Majumder S,Jaffar Ali BM, Illavazagan G, Chatterjee S. Nitric oxide/cGMP protects endothelial cells from hypoxia-mediated leakiness. Eur J Cell Biol. (2008) 87: 147-161.

[28] Majumder, S., Ilayaraja, M., Seerapu, H. R., Sinha, S., Siamwala, J. H., and Chatterjee, S. Chick embryo partial ischemia model: a new approach to study ischemia ex vivo. PLoS One (2010). 5, e10524

[29] Prozialeck WC, Edwards JR, Woods JM. The vascular endothelium as a target of cadmium toxicity.Life Sci. (2006) 79: 1493-1506.

[30] Woods JM, Leone M, Klosowska K, Lamar PC, Shaknovsky TJ, Prozialeck WC. Direct antiangiogenic actions of cadmium on human vascular endothelial cells. Toxicol In Vitro (2008) 22: 643-651.

[31] Sinha S, Sridhara SR, Srinivasan S, Muley A, Majumder S, Kuppusamy M, Gupta R, Chatterjee S NO (nitric oxide): the ring master. Eur J Cell Biol (2011) 90:58-71

[32] Fukumura, D., Gohongi, T., Kadambi, A., Izumi, Y., Ang, J., Yun, C.O., Buerk, D.G., Huang, P.L., Jain, R.K. Predominant role of endothelial nitric oxide synthasein vascular 
endothelial growth factor-induced angiogenesis and vascular permeability. Proc. Natl. Acad. U.S.A (2001) 98, 2604-2609.

[33] Ahmad, S., Hewett, P.W., Wang, P., Al-Ani, B., Cudmore, M., Fujisawa, T., Haigh,J.J., le Noble, F., Wang, L., Mukhopadhyay, D., Ahmed, A. Direct evidence for endothelial vascular endothelial growth factor receptor-1 function in nitric oxide-mediated angiogenesis. Circ. Res. (2006) 99, 715-722.

[34] Thibeault S, Rautureau Y, Oubaha M, Faubert D, Wilkes BC, Delisle C, Gratton JP. Snitrosylation of beta-catenin by eNOS-derived NO promotes VEGF-induced endothelial cell permeability.Mol Cell. (2010) 39: 468-476

[35] Baldwin, A.L., Thurston, G., Al Naemi, H. Inhibition of nitric oxide synthesis increases venular permeability and alters endothelial actin cytoskeleton. Am. J. Physiol. Heart Circ. Physiol. (1998) 274, H1776-H1784.

[36] Fulton D., Fontana J, Sowa G, Gratton J.P, M. Lin, K.X. Li, B. Michell, B.E. Kemp,D. Rodman, W.C. Sessa, Localization of endothelial nitric-oxide synthase phosphorylated on serine 1179 and nitric oxide in Golgi and plasma membrane defines the existence of two pools of active enzyme, J. Biol. Chem. 277 (2002) 4277-4284.

[37] P.W. Shaul, Regulation of endothelial nitric oxide synthase: location, Location, location, Annu. Rev. Physiol. 64 (2002) 749-774.

[38] Perumal V, Pohl S, Keane KN, Arfuso F, Newsholme P, Fox S, Dharmarajan A. Therapeutic approach to target mesothelioma cancer cells using the Wnt antagonist, secreted frizzled-related protein 4: Metabolic state of cancer cells. Exp Cell Res. (2016) 41(2):218-24.

[39] Boon, E.M., Huang, S.H., Marletta, M.A. A molecular basis for NO selectivity insoluble guanylate cyclase. Nat. Chem. Biol (2005) 1, 53-59.

[40] Roy, B., Garthwaite, J. Nitric oxide activation of guanylyl cyclase in cells revisited.Proc. Natl. Acad. U.S.A (2006) 103, 12185-12190.

[41] Pyriochou, A., Beis, D., Koika, V., Potytarcu, C., Papadimitriou, E., Zhou, Z., Papapetropoulos, A. Soluble guanylyl cyclase activation promotes angiogenesis. J. Pharmacol. Exp. Ther (2006) 319, 663-671

[42] Y. Korkmaz, The basal phosphorylation sites of endothelial nitric oxide synthase at serine (Ser)1177, Ser116, and threonine (Thr)495 in rat molar epithelial rests of malassez. J. Periodontol. 76 (2005) 1513e1519.

[43] Omori, K., Kotera, J.Overview of PDEs and their regulation. Circ. Res (2007) 100, 309327.

[44] Antonova, G., Snead, C., Antonov, A., Dimitropoulou, C., Venema, R.C., Catravas, J.D. Nitric oxide preconditioning regulates endothelial monolayer integrity via the heat shock protein 90-soluble guanylate cyclase pathway. Am J Physiol Heart Circ Physiol. (2007) 292(2):H893- 903.

[45] Majumder S, Gupta R, Reddy H, Sinha S, Muley A, Kolluru GK, Chatterjee S. Cadmium attenuates bradykinin-driven nitric oxide production by interplaying with the localization pattern of endothelial nitric oxide synthase. Biochem Cell Biol. (2009) 87: 605-620.

[46] Siamwala, J. H., Veeriah, V., Priya, M. K., Rajendran, S., Saran, U., Sinha, S., Nagarajan, S., Pradeep, T., and Chatterjee, S.. Nitric oxide rescues thalidomide mediated teratogenicity. Sci. Rep. (2012)2, 679. 
[47] Veeriah V, Saran U, Swaminathan A, Balaguru UM, Thangaraj P, Nagarajan S, Rajendran VK, Chatterjee S.Cadmium-Induced Embryopathy: Nitric Oxide Rescues Teratogenic Effects of Cadmium.Toxicol Sci. (2015) 144(1):90-104.

[48] Gertzberg, N., Neumann, P., Rizzo, V., Johnson, A. NAD(P)H oxidase mediates the endothelial barrier dysfunction induced by TNF-alpha. Am. J. Physiol. Lung Cell Mol. Physiol. (2004) 286, L37-L48.

[49] Inoue, M., Sato, E.F., Park, A.M., Nishikawa, M., Kasahara, E., Miyoshi, M., Ochi, A., Utsumi, K. Cross-talk between NO and oxyradicals, a supersystem that regulates energy metabolism and survival of animals. Free Radic. Res (2000) 33, 757-770.

[50] Suttorp, N., Hippensteil, S., Fuhrmann, M., Kru“ ll, M., Podzuweit, T. Role of nitric oxide and phosphodiesterase isoenzyme II for reduction of endothelial hyperpermeability. Am. J. Physiol. Cell Physiol (1996) 39, C778-C785.

[51] D. Longman, F.Arfuso, H.M.Viola, L.C.Hool, A.M.Dharmarajan, The role of the cysteinerich domain and netrin-like domain of secreted frizzled-related protein 4 in angiogenesis inhibition in vitro, Oncol.Res. (2012) 20;1-6.

\section{Figure Legends}

Figure 1. sFRP4 induces leakiness of the Endothelium. (A) Representative corresponding bright field and Texas red images of control and sFRP4 treated CAM. (B) Quantification of permeated dye intensity in control and $250 \mathrm{pg} / \mathrm{mlsFRP4}$ treated CAM. $\mathrm{n}=4$; ${ }^{*} \mathrm{p}<0.001$, vs. control. (C) Schematic representation of

Texas red permeability assays performed in vitro on EC monolayers. (D) Graphical representation of Texas red permeability assay performed on EC monolayers following treatment with 125, 250 and 500 $\mathrm{pg} / \mathrm{ml}$ doses of sFRP4. $\mathrm{n}=3$; ${ }^{*} \mathrm{p}<0.001$, vs. control. Plotting of $250 \mathrm{pg} / \mathrm{ml}$ sFRP4 treatment induced dye permeation on Texas Red-Cadmium scale in CAM (E) and endothelial monolayers (F) equivalent to $5 \mu \mathrm{M}$ $\mathrm{CdCl}_{2}$. (G) Representative images of phallodin stained monolayers and single cells in control and sFRP4 treated cells. Values represent the mean for each group, obtained from 3 independent experiments \pm SEM (one way ANOVA and LSD).

Figure 2. sFRP4 dampens NO production of EC. (A) Graph representing estimated NO levels of EC subjected to 125, 250 and $500 \mathrm{pg} / \mathrm{ml}$ doses of sFRP4. $\mathrm{n}=6$; ${ }^{*} \mathrm{p}<0.01$, vs. control; ${ }^{* *} \mathrm{p}<0.001$, vs. control. (B) Time-dependent study demonstrated that $250 \mathrm{pg} / \mathrm{ml}$ sFRP4 suppressed NO production from 3 h upto 24 h post treatment. $\mathrm{n}=6$; $\dagger \mathrm{P}<0.002$, 6h sFRP4 vs. control; ${ }^{*} \mathrm{p}<0.01$, $12 \mathrm{~h}$ sFRP4 vs. control; ${ }^{* *} \mathrm{p}<0.001$, 24h sFRP4 vs. control. (C) Representative corresponding bright field and DAF-2DA images of control and sFRP4 treated HDMVEC and EAhy926 cells. (D) Graphical representation of DAF-2DA fluorescence intensity calculated. $\mathrm{n}=4$; $\dagger \dagger \mathrm{P}<0.003$, vs. control; ${ }^{* *} \mathrm{p}<0.001$, vs. control. (E) Graphical representation of NO production quantified for bovine aortic cells following treatment. $\mathrm{n}=3 ;{ }^{* *} \mathrm{p}<0.001$, 
vs. control. Values represent the mean for each group, obtained from 3 independent experiments \pm SEM (one way ANOVA and LSD).

Figure 3. sFRP4 blunts NO in cGMP dependent manner. (A) Representative images of eNOS localization of ECV304 cells stably transfected with eNOS-GFP following treatment with (i) control, (ii) BK, (iii) sFRP4 and (iv) sFRP4 + BK. (B) Graphical representation of the number of cells demonstrating eNOS localization. (C) Western blot of triton-soluble and insoluble proteins probed for eNOS in control (c) and sFRP4 (s4) treated EAhy926 cells. (D) Representative corresponding bright field and FlincG transfected EAhy926 cells following treatment with control, negative control cPTIO and sFRP4. (E) Graphical representation of FlincG fluorescence intensity calculated following treatments. $\mathrm{n}=4 ;{ }^{* *} \mathrm{p}<0.001$, vs. control. Values represent the mean for each group \pm SEM (one way ANOVA and LSD).

Figure 4. (A) Graphical representation of 2 and $4 \mathrm{~h}$ scratch-wound healing results following treatment with control, sFRP4, YC1, SC, 8-Br and combinations. $\mathrm{n}=4$; \#p<0.04, 2h sFRP4 + 8-Br vs. sFRP4; ${ }^{*} \mathrm{p}<0.01$, 4h sFRP4 $+8-\mathrm{Br}$ vs. sFRP4. (B) Addition of SP and 8-Br alleviated sFRP4 mediated suppression of NO production. $\mathrm{n}=6$; ${ }^{* *} \mathrm{p}<0.001$, vs. sFRP4. (C) Estimation of NO production following addition of anti-oxidants CAT and MnTBAP respectively to sFRP4 treated cells. Values represent the mean for each group \pm SEM (one way ANOVA and LSD).

Figure 5. Supplementation of NO or neutralization of redox levels rescued EC from sFRP4 induced oxidative stress. Graphs represent endothelial ROS levels evaluated following treatment with control, sFRP4, SP, 8-Br, CAT, MnTBAP and their combinations. $n=6$. (A) Total ROS levels quantitated following treatment of EC. $\dagger \mathrm{P}<0.006$, ${ }^{* *} \mathrm{p}<0.001,{ }^{*} \mathrm{p}<0.01$; vs. sFRP4. (B) Hydrogen peroxide levels quantitated for EC following treatment. ${ }^{* *} \mathrm{p}<0.001$, ${ }^{*} \mathrm{p}<0.01$, $\# \mathrm{p}<0.02$, vs. sFRP4. (C) Superoxide levels quantitated following treatment. ${ }^{* *} \mathrm{p}<0.001,{ }^{*} \mathrm{p}<0.01$, vs. sFRP4. (D) Peroxynitrite levels quantitated following treatment. ${ }^{* *} \mathrm{p}<0.001,{ }^{*} \mathrm{p}<0.01$, vs. sFRP4. Values represent the mean for each group \pm SEM (one way ANOVA and LSD).

Figure 6. Supplementation of NO or neutralization of redox levels rescued EC from sFRP4 induced apoptosis. (A) Representative images of corresponding bright field, Annexin V, and PI of endothelial cells following treatment with control, sFRP4, SP, 8-Br, CAT, MnTBAP and their combinations. Staurosporine (STP) was used as positive control. (B) Graphical representation of the intensity of Annexin V and PI fluorescence calculated. $\mathrm{n}=6$; ${ }^{*} \mathrm{p}<0.001,{ }^{*} \mathrm{p}<0.01$, vs. sFRP4 - Annexin V. $\dagger \mathrm{p}<0.01$, \# $\mathrm{p}<0.03$, vs sFRP4 - PI. Values represent the mean for each group \pm SEM (one way ANOVA and LSD). 
Figure 7. (A) Representative corresponding bright field, eNOS, DAR-4M-AM and merged images of control and sFRP4 treated ECV304 stably transfected with eNOS-GFP monolayers. Black arrows indicate formations of gaps in EC monolayer. (B) Graph comparing the cell clusters demonstrating green (eNOS), red (DAR-4M-AM), yellow (merged) and black (gaps) in control and sFRP4 treated cells. (C) Graphs representing the fluorescence intensity calculated for eNOS and DAR-4M-AM in control and sFRP4 treated monolayers. ${ }^{* *} \mathrm{p}<0.001$, vs. control - eNOS; $\dagger \mathrm{p}<0.001$, vs. control - DAR-4M-AM. Values represent the mean for each group \pm SEM (one way ANOVA and LSD).

Figure 8. (A) Representative corresponding bright field, Texas red, Annexin V and merged images of control, sFRP4, SP and sFRP4 + SP treated CAM. (B) Graph representing the Texas red and Annexin V fluorescence intensity calculated. ${ }^{* *} \mathrm{p}<0.001$, vs. control - Texas red; $\dagger \mathrm{p}<0.001$, vs. control - Annexin V. (C) Quantification of permeated texas red dye intensity in control, sFRP4, SP and sFRP4 + SP treated CAM. ${ }^{* *} \mathrm{p}<0.001$, vs. sFRP4. (D) Graphical representation of Texas red permeability assay performed on EC monolayers following treatment with control, sFRP4, SP, 8-Br, CAT, MnTBAP and their combinations. $\dagger^{\dagger} \mathrm{p}<0.002,{ }^{*} \mathrm{p}<0.01$ vs. sFRP4. Values represent the mean for each group \pm SEM (one way ANOVA and LSD).

Figure 9. (A) Graph representing the estimated NO levels of EC subjected control, sFRP4 and sFRP4 CRD domain peptides. $\dagger \mathrm{p}<0.002,{ }^{* *} \mathrm{p}<0.001$, vs. control. (B) Quantification of permeated texas red dye intensity in control, sFRP4 and sFRP4 CRD peptides (CP-133-10 and CP-134-10). \#p<0.005, ${ }^{* *} \mathrm{p}<0.001$,vs control. (C) Representative images of corresponding bright field, Annexin V, PI stained cells following treatment with CP-133-10 and CP-134-10. (D) Graphical representation of the intensity of Annexin V and PI fluorescence calculated. $n=6$. Values represent the mean for each group \pm SEM (one way ANOVA and LSD). 